\title{
Concentration fluctuations of large Stokes number particles in a one-dimensional random velocity field
}

\author{
Piero Olla ${ }^{1}$ and M. Raffaella Vuolo ${ }^{2}$ \\ ${ }^{1}$ ISAC-CNR, and INFN Sez. Cagliari, I-09042 Monserrato, Italy. \\ ${ }^{2}$ Dipartimento di Fisica and INFN, Universitá di Cagliari, I-09042 Monserrato, Italy.
}

(Dated: July 22, 2018)

\begin{abstract}
We analyze the behavior of an ensemble of inertial particles in a one-dimensional smooth Gaussian velocity field, in the limit of large inertia, but considering a finite correlation time for the random field. The amplitude of the concentration fluctuations is characterized by slow decay at large inertia and a much larger correlation length than that of the random field. The fluctuation structure in velocity space is very different from predictions from short-time correlated random velocity fields, with only few particle pairs crossing at sufficiently small relative velocity to produce correlations. Concentration fluctuations are associated with depletion of the relative velocity variance of colliding particles.
\end{abstract}

PACS numbers: 05.10.Gg, 05.40.-a, 46.65.+g, 47.27.T-

\section{INTRODUCTION}

Starting from the work of Deutsch [1], it has been known for some time now, that inertial particles in a random velocity field undergo clustering phenomena. These behaviors are observed in numerical simulation of turbulence as well (see e.g [2] and references therein) and are thought to give an important contribution to coalescence phenomena, e.g. in the process of rain formation 3, 44. Both in the case of random fields and of real turbulence, spatial inhomogeneity of the statistics, though contributing to particle segregation [5], does not appear to be an essential factor.

Random velocity fields with various statistical properties, have been used to model inertial particles in turbulent flows, both in the presence of gravity [6, 7] and in its absence 8, 9]. Simplified models disregarding in part or in total the spatial structure of the velocity field have been introduced as well (see e.g. [10, 11]), to cope with the difficulty of the analytical treatment of the multiparticle statistics.

In the simplest instance, an inertial particle in a turbulent flow is characterized by the relaxation time of its velocity relative to the fluid: the Stokes time $\tau_{S}=$ $2 / 9 a^{2} \lambda / \nu_{0}$, where $a$ is the particle radius, $\lambda$ is the ratio of the particle to fluid density (assumed large) and $\nu_{0}$ is the kinematic viscosity of the fluid [12]. Experimental data [13 and numerical simulations 14 both indicate that clustering is stronger for particles with $\tau_{S}$ of the order of the Kolmogorov time scale. This observation, and the fact that Stokes times for most atmospheric aerosols of interest lie in this range [3] have motivated substantial analytical effort in the small Stokes time regime, with smooth velocity fields mimicking turbulence at the Kolmogorov scale [4, 15].

Some analysis has been carried on also for Stokes times in the turbulent inertial range [8, 16, 17], but numerical simulations [18] indicate that concentration fluctuations do not follow simple scaling rules.

Due to the relative ease of analytical treatment, an- other regime which attracted attention is that of inertial particles in a smooth random velocity field, with correlation time $\tau_{E}$ much shorter than the geometric scale $r_{\mathrm{v}} / \sigma_{u}$ obtained from the correlation length $r_{\mathrm{v}}$ and the amplitude $\sigma_{u}$ of the velocity field fluctuations [19, 20]. However, this is a short-time correlated regime that is very different from the situation in realistic turbulent flows: $\tau_{E} \sigma_{u} / r_{\mathrm{v}}=O(1)$.

The analysis of random velocity field models has allowed to identify at least two clustering mechanisms, expected to be present also in real turbulence. The first one, originally proposed in [6], is preferential concentration of heavy (light) particles in the strain (vortical) regions of the flow. This effect has been recently observed also in the case of particles with very small inertia [22, 23]. The second mechanism, already present in one dimension (1D) 21, 24], is that of the particles catching one another in their motion, as they slip with respect to the fluid. In the case of smooth velocity fields, this leads to the formation of caustics in the instantaneous concentration field, which, in turn, act as back-bones for the clustering process 25]. The clustering itself is a long-time process, which can be described in terms of the Lyapunov exponents of the particle pair dynamics [25, 26].

Focusing on the second mechanism, it appears that caustics formation tends to become maximum for $\tau_{S} / \tau_{E}, \tau_{E} \sigma_{u} / r_{\mathrm{v}} \rightarrow 1$ [27], and that, at the same time, clustering becomes weaker. For larger $\tau_{S}$, one expects that the particles be scattered by the velocity fluctuations they cross in their motion [28] as if undergoing Brownian diffusion, resulting in vanishing particle correlations and in clustering destruction. It is to be stressed that the interest of this limit is by no means academic, as particles in turbulent flows for which $\tau_{S}$ lies in the inertial range (or above), will see smaller vortices precisely in this way.

Purpose of this paper is to understand in detail how and under which conditions the uncorrelated limit described in [28] is achieved. It will appear that clustering destruction occurs in a very non trivial way, requiring 
consideration, among the other things, of how particle correlations decay at scales comparable with or above $r_{\mathrm{v}}$. We are going to show that, provided we are away from the short correlation time regime $\tau_{E} \ll \sigma_{u} / r_{\mathrm{v}}$, clustering at $\tau_{S} \gg \tau_{E}$ will not be dominated by the smallseparation particle pair dynamics (small with respect to $r_{\mathrm{v}}$ ) described in [21]. Particle pairs remaining close long enough for a Lyapunov exponent approach to be appropriate, are still present, but their contribution to concentration fluctuations is negligible.

The key mechanism for the destruction of concentration fluctuations will appear to be that, at large $\tau_{S} / \tau_{E}$ and finite $\tau_{E} \sigma_{u} / r_{\mathrm{v}}$, only particles with increasingly small relative velocities (but not small enough for a local theory on the lines of $[21,26]$ to be valid) have a chance to be correlated. This is to be contrasted with the picture in [27], of correlation fluctuations disappearing as caustics occupy in the above limit larger and larger portions of space, and with the one in [26] of saturation to the space dimension of the particle distribution correlation dimension. Neither in [26] nor in [27], however, was a quantitative prediction on clustering decrease provided.

The present analysis will also allow to show that, for large $\tau_{S} / \tau_{E}$ and rather generic (not too large) values of $\sigma_{u} \tau_{E} / r_{\mathrm{v}}$, clustering is associated with smaller typical relative inter-particle velocities, compared to what would be observed in the absence of correlations. Thus, there are circumstances under which, clustering may hinder rather than enhance coalescence phenomena.

This paper is organized as follows. The main definitions and approximations will be presented in Section II, following by and large the notation of [19, 20, 21]. In Section III, the pair particle dynamics will be analyzed in the large $\tau_{S}$ limit, identifying the relevant time and velocity scales for clustering. In Section IV, the relation between particle-particle velocity depletion and clustering will be established, and heuristic estimates for the concentration correlation will be obtained, starting from the Fokker-Planck equation for the distribution of the relative particle velocity and coordinate. In Section V, a proof for the dominance of slowly approaching particle pairs in cluster generation will be provided. Section VI contains the conclusion.

\section{MODEL EQUATIONS}

Consider an ensemble of inertial particles transported by a $1 \mathrm{D}$ zero mean, Gaussian random velocity field $u(x, t)$ with correlation

$$
\langle u(x, t) u(0,0)\rangle=\sigma_{u}^{2} F\left(t / \tau_{E}\right) g\left(x / r_{\mathrm{v}}\right),
$$

where $g(0)=F(0)=1$, the function $g$ is assumed to be smooth and to have all necessary moments, and $\int_{0}^{\infty} F(t) \mathrm{d} t=1$. The particles are immaterial, so that they can cross without interaction, and their velocity $v$ is taken to obey the Stokes equation:

$$
\dot{v}=\tau_{S}^{-1}[-v(t)+u(x(t), t)] .
$$

Introduce the Stokes and Kubo numbers:

$$
S=\tau_{S} / \tau_{E}, \quad K=\sigma_{u} \tau_{E} / r_{\mathrm{v}}
$$

and choose units so that $\sigma_{u}=\tau_{S}=1$; therefore:

$$
\tau_{E}=S^{-1}, \quad r_{\mathrm{v}}=(K S)^{-1} .
$$

In the regime $S \gg 1$, the particle velocity will change little on the lifetime of a fluid fluctuation and Eq. (2) could be approximated by a Langevin equation. This, independently of $K$, i.e. of the random field being shorttime correlated or not. It is possible to substitute into Eq. (2):

$$
u(x(t), t) \rightarrow\left(2 \tau_{p}(v)\right)^{1 / 2} \xi(t),
$$

with $\xi(t)$ white noise: $\langle\xi(t) \xi(0)\rangle=\delta(t)$ and

$$
\tau_{p}(v)=\int_{0}^{\infty} \frac{\langle u(x(t), t) u(x(0), 0) \mid v\rangle}{\left\langle u^{2}(x(t), t)\right\rangle} \mathrm{d} t
$$

the correlation time of the fluid velocity sampled by a particle moving at speed $v$. In the above formula, the averages are calculated along trajectories, with $\langle. \mid v\rangle$ indicating the condition on $v$. The average on the trajectory in the denominator, due precisely to the presence of concentration fluctuations, does not necessarily coincide with the space average $\left\langle u^{2}\right\rangle \equiv \sigma_{u}^{2}$.

From Eq. (11), we can put $\tau_{p} \simeq \tau_{E}$, provided $r_{\mathrm{v}} / \sigma_{v} \gg$ $\tau_{E}$, with $\sigma_{v}^{2}$ the particle velocity variance. If $\tau_{p} \simeq \tau_{E}$, we can estimate from Eqs. (2) and (5):

$$
\sigma_{v}^{2} \simeq S^{-1}
$$

which is satisfied if $K^{2} \ll S$. Following the same line of reasoning (see Appendix), it is possible to show that prescription problems in the definition of the white noise arise only at $O\left(K^{2} / S\right)$. Notice that $K^{2} \ll S$ and $S \gg$ 1 imply $\chi=K / S \ll 1$, where $\chi$ is precisely the force magnitude parameter introduced in [21]. This condition is easily satisfied unless one chooses to work in a frozen turbulence regime $K \gg 1$.

Turning to the relative motion of particle pairs, let us introduce difference variables

$$
\nu=v_{2}-v_{1}, \quad r=x_{2}-x_{1},
$$

where 1 and 2 label members of a particle pair, and indicate $u_{1,2}(t)=u\left(x_{1,2}(t), t\right)$. In the regime $S \gg 1$, starting from Eq. (2), we can approximate the equation for the relative motion of particles with the Langevin equation:

$$
\dot{\nu}=-\nu+b(r) \xi, \quad \dot{r}=\nu .
$$

For $S \gg K^{2}$, we have for the noise square amplitude $B(r)=b^{2}(r)$, from Eq. (1):

$$
\begin{gathered}
B(r)=\int_{-\infty}^{\infty}\left\langle\left[u_{2}(t)-u_{1}(t)\right]\left[u_{2}(0)-u_{1}(0)\right] \mid \nu, r\right\rangle \mathrm{d} t \\
\simeq 4 S^{-1}\left[1-g\left(r / r_{\mathrm{v}}\right)\right] .
\end{gathered}
$$

The decoupling of the difference variables $(\nu, r)$ from the center of mass variables $\frac{1}{2}\left(x_{1}+x_{2}\right)$ and $\frac{1}{2}\left(v_{1}+v_{2}\right)$ descends basically from the condition $\tau_{p}(v) \simeq \tau_{E}$. 


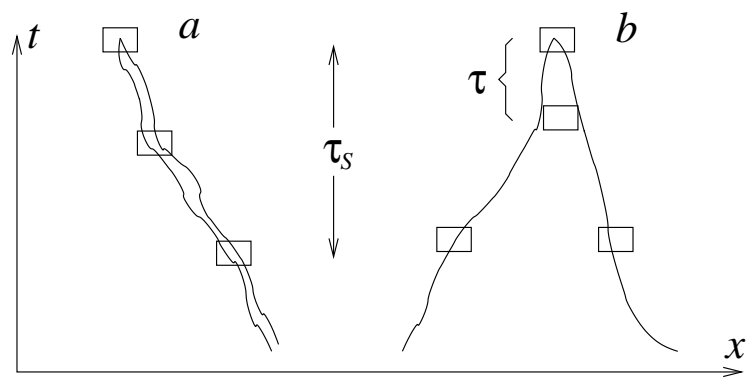

FIG. 1: Typical particle pair trajectories for $\epsilon \ll 1(a)$ and $\epsilon \gg 1(b)$. The exit time to the right is $\tau \sim \epsilon^{-1 / 2} \tau_{S}$. The small rectangles are space-time correlated regions for the fluid, of size $r_{\mathrm{v}}$ and duration $\tau_{E}$. The time $\tau_{S}$ quantifies the particle memory. The particle approach in $(a)$ is governed by an $O(\epsilon)$ negative Lyapunov exponent [21].

\section{TWO-PARTICLE DYNAMICS}

In order to determine how concentration fluctuations are generated, we need to understand the particle pair dynamics at small separations. In the large $S$ limit, we expect that the velocities of colliding particles be uncorrelated, however, this condition turns out not to be automatically satisfied. A condition for the particles to be weakly correlated, despite being close, is that the Stokes time be much longer than the time spent at $|r| \lesssim r_{\mathrm{v}}$, so that the velocity of the two particles will be the result of many contributions by uncorrelated regions of the fluid (see Fig. 1). Under these conditions, particles will move ballistically at scale $|r| \lesssim r_{\mathrm{v}}$, and they will cross at velocity $\nu \sim \sigma_{v}=S^{-1 / 2}$, after a time $\tau \sim r_{\mathrm{v}} / \sigma_{v} \sim\left(K^{2} S\right)^{-1 / 2}$. The weak correlation condition is therefore $r_{\mathrm{v}} / \sigma_{v} \ll \tau_{S}$, i.e.:

$$
\epsilon=K^{2} S \gg 1
$$

The parameter $\epsilon$ is the same as in the theory in [19, 20], who considered in detail the small $\epsilon$ regime. For $\epsilon \ll 1$, the time spent by a pair of particles at $|r| \lesssim r_{\mathrm{v}}$ before collision, would be longer than $\tau_{S}$, and the particles would have memory only of that last portion of their history, when they were at $|r| \ll r_{\mathrm{v}}$; therefore, $\nu$ could not be treated, at the time of crossing, as a difference of uncorrelated velocities [29].

Notice that the conditions $\epsilon \ll 1$ and $S \gg 1$ can be realized only if $K \ll S^{-1 / 2} \ll 1$, corresponding to a short-time correlated random velocity field. In this regime, $\epsilon$ plays the role of an effective Stokes number (see in particular [26]), in which the place of $\tau_{E}$ is taken by $r_{\mathrm{v}} /\left(\sigma_{u}^{2} \tau_{E}\right)$, that is the diffusion time of a (non-inertial) tracer across a distance $r_{\mathrm{v}}$. Notice also that the condition $\tau_{S} \gg \tau_{E}, r_{\mathrm{v}} / \sigma_{u}$, i.e. $K S, S \gg 1$, is not sufficient by itself to guarantee weak correlation, that in fact is not fulfilled in the range $S^{-1} \ll K \ll S^{-1 / 2}$, where $\epsilon \ll 1$.

Back to the large $\epsilon$ range, although most particles will be weakly correlated, those approaching at speed $|\nu| \ll \sigma_{v}$ may stay at $|r|<r_{\mathrm{v}}$ sufficiently long to end up being strongly correlated. It is then important to understand whether an effect like clustering, which depends on pair-correlation, is due to the effect of the many weakly correlated particle pairs, or to the one of the few strongly correlated ones.

We will answer this question in Sec. V. For the moment, we identify the important time and velocity scales involved in the dynamics of slowly approaching particle pairs. We rescale variables:

$$
\hat{t}=t / t_{\mathrm{v}}, \quad \hat{\nu}=\nu / \nu_{\mathrm{v}}, \quad \hat{r}=r / r_{\mathrm{v}},
$$

where

$$
t_{\mathrm{v}}=(2 \epsilon)^{-1 / 3}, \quad \nu_{\mathrm{v}}=2^{1 / 3} \epsilon^{-1 / 6} \sigma_{v}
$$

and $r_{\mathrm{v}}$ is given in Eq. (44). In the new variables, at short separations, the noise amplitude (9) can be Taylor expanded and Eq. (8) becomes:

$$
\dot{\hat{\nu}}=-(2 \epsilon)^{-1 / 3} \hat{\nu}+\hat{r} \xi ; \quad \dot{\hat{r}}=\hat{\nu} .
$$

Thus, $t_{\mathrm{v}}$ and $\nu_{\mathrm{v}}$ are in some sense the time and velocity scales at the correlated scale $r_{\mathrm{v}}$. Notice that the condition for Eq. (13) to be meaningful as a stochastic differential equation is $t_{\mathrm{v}} \gg \tau_{E}$, i.e. again $\chi=K / S \ll 1$.

To understand better the meaning of $t_{\mathrm{v}}$ and $\nu_{\mathrm{v}}$, consider the separation of a pair of particles such that $\hat{r}(0)=0$ and $|\hat{\nu}(0)| \ll 1$. This is equivalent to looking at the approach of a particle pair, with conditions imposed on the velocity at the moment of crossing, rather than at a previous initial time.

In the initial phase, the separating particles will move at almost constant relative velocity, and $\hat{r} \simeq \hat{\nu}(0) \hat{t}$. The variation of $\hat{\nu}$ will be $\Delta \hat{\nu} \simeq \hat{\nu}(0) \int_{0}^{\hat{t}} \hat{t}^{\prime} \xi\left(\hat{t}^{\prime}\right) \mathrm{d} \hat{t}^{\prime}$ so that $\left\langle\Delta \hat{\nu}^{2}\right\rangle \simeq \hat{\nu}^{2}(0) \hat{t}^{3} / 3$. The initial phase will last until $\Delta \hat{\nu} \sim \hat{\nu}(0)$, i.e. until $\hat{t} \sim 1$, when $\hat{r} \sim \hat{\nu}(0)$. We then see that $\hat{\nu} \sim 1$, i.e. $\nu=\nu_{\mathrm{v}}$ is the minimum relative velocity to which the approximation of constant relative velocity at the correlated scale applies, and $t_{\mathrm{v}}$ is the associated exit time: $\left|r\left(t_{\mathrm{v}}\right)\right| \sim r_{\mathrm{v}}$.

The following phase will be determined by the linear nature of Eq. (13); for $t<\tau_{S}$, the drift $-(2 \epsilon)^{-1 / 3} \hat{\nu}$ can still be disregarded, and we expect $\hat{\nu}(\hat{t}), \hat{r}(\hat{t}) \sim$ $\hat{\nu}(0) \exp (\hat{t})$. After a time of the order of $-\ln \hat{\nu}(0)$, the particles will have moved apart by $\hat{r} \sim 1$, i.e. they will be moving out of a correlated region for $u$. In this final part of the separation process, Eq. (13) will cease to be valid, with $\hat{\nu}$ obeying instead $\dot{\hat{\nu}} \simeq \xi$. For $\hat{\tau}_{S} \gg \hat{t} \gg-\ln \hat{\nu}(0), \nu$ will behave like a Brownian motion: $\hat{\nu}(\hat{t}) \sim \hat{t}^{1 / 2}$, while $\hat{r}$ will undergo a Richardson-like diffusion process: $\hat{r}(\hat{t}) \sim \hat{t}^{3 / 2}$.

In conclusion, we have two groups of particle pairs.

1. The great majority, for which, at the moment of crossing: $\nu \sim \sigma_{v}$; their motion in a correlated region can be approximated as ballistic and their permanence time is $\tau \sim \epsilon^{-1 / 2} \tau_{S}$. 
2. A smaller fraction, for which, at the moment of crossing: $|\nu| \lesssim \nu_{\mathrm{v}}$, with $\nu_{\mathrm{v}} \sim \epsilon^{-1 / 6} \sigma_{v}$; their dynamics in a correlated region is determined by fluctuations of $u(x, t)$ at scale $\lesssim r_{\mathrm{v}}$ and their permanence time grows logarithmically as $\nu \rightarrow 0$ : $\tau(\nu) \sim \epsilon^{-1 / 3} \ln \left(\nu_{\mathrm{v}} /|\nu|\right)$.

The fact that for $\epsilon \gg 1, t_{\mathrm{v}} \ll \tau_{S}$, has the consequence that only particles meeting at exponentially small relative velocities

$$
\nu_{\tau} \sim \sigma_{v} \epsilon^{-1 / 6} \exp \left(- \text { const. } \epsilon^{1 / 3} \tau / \tau_{S}\right),
$$

will have a chance to stay within a correlated region for a time $\tau \gtrsim \tau_{S}$. An exponentially low level of molecular diffusion is thus sufficient to destroy these pairs. Even in the absence of molecular diffusion, however, the relative motion of these pairs is characterized in the limit $\tau / \tau_{S} \rightarrow \infty$ by a positive Lyapunov exponent [21] and no long-time contributions to clustering are present. The remaining particle pairs can be shown not to produce singular contributions to the two-particle probability density function (PDF) for $\nu$ [34]. We can then estimate the fraction of particle pairs staying close for a time $\tau$, i.e. the fraction of particles crossing at speed $\nu_{\tau}$ (provided of course $\left.\tau>t_{\mathrm{v}}\right)$, as

$$
\nu_{\tau} / \sigma_{v} \sim \epsilon^{-1 / 6} \exp \left(- \text { const. } \epsilon^{1 / 3} \tau / \tau_{S}\right) .
$$

This, again, is exponentially small for $\tau \gtrsim \tau_{S}$ and large $\epsilon$, while the fraction of particle pairs that are merely not ballistic, is the much larger value $\nu_{\mathrm{v}} / \sigma_{v} \sim \epsilon^{-1 / 6}$.

The situation in the small $\epsilon$ regime is very different, as Eq. (13) leads to instantaneous equilibration of the particle velocity: $\left\langle\nu^{2} \mid r\right\rangle \sim \sigma_{v}^{2}\left(r / r_{v}\right)^{2}$ and the ballistic particle fraction, associated with caustics formation, disappears [21, 27, 30]. Hence, $\sigma_{v}^{-2}\left\langle\nu^{2} \mid r=0\right\rangle \rightarrow 0$ and the particles behave for $\epsilon \ll 1$ as a mono-disperse phase (although with velocity not locally equal to that of the fluid, as it happens instead in the small $S$ limit [15]). At $\epsilon \rightarrow 0$, all particle pairs are therefore co-moving.

\section{THE PAIR DISTRIBUTION}

We have seen at the end of Section II that in the regime $S \gg 1, S \gg K^{2}$, the difference variables $\nu, r$ decouple from the center of mass ones. This means that at equilibrium, the two-particle PDF will be in the form

$$
\rho\left(v_{1,2}, x_{1,2}\right)=\Omega^{-1} \rho\left(\frac{v_{1}+v_{2}}{2}\right) \rho(\nu, r),
$$

where $\Omega$ is the length of the domain for $x_{1,2}$. (Unless ambiguous, we do not use subscripts to identify PDF's referring to different stochastic variables). Multiplying by $N^{2}$, with $N$ the total number of particles in the domain $\Omega$ and integrating over $\mathrm{d} v_{1} \mathrm{~d} v_{2}$, we obtain the expression for the concentration correlation:

$$
\langle n(r) n(0)\rangle=\bar{n}^{2} \Omega \int \mathrm{d} \nu \rho(\nu, r):=\bar{n}^{2}[1+f(r)],
$$

where $\bar{n}=N / \Omega$ is the mean concentration. The quantity $f(r)=\Omega \rho(r)-1$ gives the strength of the concentration fluctuations.

The difficulty of the problem, compared with the $\epsilon \ll 1$ limit, is the finite width in $\nu$ of the $\operatorname{PDF} \rho(\nu, r)$. In the $\epsilon \ll 1$ limit, small $r$ implied small $\nu$, a long permanence at that particular $r$ and the possibility of linearizing the Fokker-Planck equation in $r$ along the lines of [21, 31]. In our case this is not possible, and we must consider the full Fokker-Planck equation, which, from Eqs. (8) and (9), reads, at steady state:

$$
\nu \partial_{r} \rho(\nu, r)-\partial_{\nu}[\nu \rho(\nu, r)]=\frac{1}{2} B(r) \partial_{\nu}^{2} \rho(\nu, r)
$$

(see [32] for another example of kinetic treatment of the particle pair statistics in turbulent flows).

Taking moments of the Fokker-Planck equation (15) allows to draw important conclusions on the connection between clustering and decrease of the relative velocity variance. The first two moments of Eq. (15) read:

$$
\begin{aligned}
& \partial_{r}(\langle\nu \mid r\rangle \rho(r))=0, \\
& \partial_{r}\left(\left\langle\nu^{2} \mid r\right\rangle \rho(r)\right)+\langle\nu \mid r\rangle \rho(r)=0,
\end{aligned}
$$

where $\left\langle\nu^{p} \mid r\right\rangle \rho(r)=\int \mathrm{d} \nu \nu^{p} \rho(\nu, r)$. We can impose boundary conditions to Eq. (16) at $r \rightarrow \infty$, where the solution, corresponding to uncorrelated particles, is known. The first moment equation tells us that $\langle\nu \mid r\rangle \rho(r)$ is constant; but, at $|r| \gg r_{\mathrm{v}}$ correlations are absent; therefore $\rho(r \rightarrow \infty)=\Omega^{-1}$ and $\langle\nu \mid \infty\rangle=0$; thus $\langle\nu \mid r\rangle \rho(r)=0$ for all $r$. Substituting into the second one, we get $\partial_{r}\left(\left\langle\nu^{2} \mid r\right\rangle \rho(r)\right)=0$, and, using again $\rho(r \rightarrow \infty)=\Omega^{-1}$ and $\left\langle\nu^{2} \mid \infty\right\rangle:=\sigma_{\nu}^{2}=2 S^{-1}$, we get:

$$
\sigma_{\nu}^{-2}\left\langle\nu^{2} \mid r\right\rangle=[1+f(r)]^{-1} .
$$

In other words, clustering in 1D is necessarily associated with velocity variance decrease. Notice that this result rests only on the applicability of the Fokker-Planck equation (15), which requires $S \gg 1, \chi=K / S \ll 1$, but is otherwise independent of $\epsilon$.

An idea of how $f(r)$ goes to zero as $\epsilon \rightarrow \infty$ can be obtained treating the function $g$ in Eq. (9) as a perturbation. This is motivated by the observation (see previous section) that most particles spend very little time at distances $|r|<r_{\mathrm{v}}$, and by the assumption (verified in [34]) that the remaining particles produce only a correction to $\rho(r)$. To lowest order, the particles are therefore independent random walkers with velocity correlation time $\tau_{S}$. A further simplification is obtained assuming that the scale of variation of $\rho(\nu, r)$ remains $\sigma_{v}=S^{-1 / 2}$, as in the unperturbed case. We can then write $\rho \simeq \rho_{0}+\rho_{1}$, with

$$
\rho_{0}(\nu, r)=\Omega^{-1} \rho_{0}(\nu)=\frac{S^{1 / 2}}{2 \pi^{1 / 2} \Omega} \exp \left(-\frac{S \nu^{2}}{4}\right)
$$

the solution of Eq. 115) with $g\left(r / r_{\mathrm{v}}\right)$ set to zero, and estimate $\nu \partial_{r} \rho_{1} \sim S^{-1 / 2} \partial_{r} \rho_{1}, \partial_{\nu}\left(\nu \rho_{1}\right)-(1 / 2) B_{0} \partial_{\nu}^{2} \rho_{1} \sim$ $\Gamma \rho_{1}$ for some constant $\Gamma$. Substituting into Eq. (15):

$$
\left[S^{-1 / 2} \partial_{r}+\Gamma\right] \rho_{1} \sim B_{0} g\left(r / r_{\mathrm{v}}\right) \rho_{0}
$$


A new length $S^{-1 / 2}$, the distance travelled by a particle in a Stokes time, thus enters the problem. On the scale of $S^{-1 / 2}$, the term $g$ in Eq. (19) behaves like a Dirac delta and we find:

$$
\rho_{1}(\nu, r) \sim \Omega^{-1} \epsilon^{-1 / 2} \exp \left(-\Gamma S^{1 / 2}|r|\right) \rho_{0}(\nu) .
$$

Substituting into Eq. (14) would lead to concentration fluctuations with $O\left(\epsilon^{-1 / 2}\right)$ variance and $O\left(S^{-1 / 2}\right)$ correlation length.

\section{THE VELOCITY STRUCTURE}

The analysis carried on in the previous section did not answer the question of which particle pairs are responsible for the production of the concentration fluctuations at large $\epsilon$. We are going to show here that concentration fluctuations are indeed produced by slowly approching particle pairs.

We prove that the contrary is impossible and assume therefore that the dominant contribution is due to particles approaching ballistically at scale $r_{\mathrm{v}}$, which spend a time $\tau \sim \epsilon^{-1 / 2} \tau_{S}$ at that scale. In order to show that the assumption of dominance of ballistic particles cannot be true, it is sufficient to calculate $\rho(r)=\int \rho(\nu, r) \mathrm{d} \nu$ as the stationary solution of the evolution equation

$$
\rho(r ; 0)=\int \mathrm{d} r^{\prime} \mathrm{d} \nu^{\prime} \rho\left(r ; 0 \mid \nu^{\prime}, r^{\prime} ;-T\right) \rho\left(\nu^{\prime}, r^{\prime} ;-T\right),
$$

where $\rho(\nu, r ; t)$ is a generic non-equilibrium $\mathrm{PDF}$ and $\rho\left(r ; 0 \mid \nu^{\prime}, r^{\prime} ;-T\right) \equiv \rho\left(r(0)=r \mid \nu(-T)=\nu^{\prime}, r(-T)=r^{\prime}\right)$ is a transition PDF for the dynamics described by Eqs. 88 9). If we take $\tau \ll T \ll \tau_{S}$ and $|r| \lesssim r_{\mathrm{v}}$, for most particles [i.e. for typical values of $\nu(-T)$ ], we will have:

$$
|r(-T)| \simeq|r(0)-\nu(-T) T| \gg r_{\mathrm{v}},
$$

which descends from the fact that, for $T \ll \tau_{S}$ and $\nu \sim$ $\sigma_{v}, \nu$ can be approximated as constant. But Eq. (22) tells us that the dominant contribution to the integral in Eq. (21) for $\rho(r ; 0)=\rho(r)$ (the equilibrium PDF), is from values of $r^{\prime}$ for which $\rho\left(\nu^{\prime}, r^{\prime},-T\right)=\rho\left(\nu^{\prime}, r^{\prime}\right) \simeq \rho_{0}\left(\nu^{\prime}, r^{\prime}\right)$, where $\rho_{0}$ is the spatially homogeneous PDF of Eq. (18). For the ballistic particle pairs (which we are assuming to dominate the statistics), we can calculate the correction to ballistic motion, writing $r(t)=r_{0}(t)+r_{1}(t)$, where, using Eq. (8):

$$
\begin{aligned}
& r_{0}(t)=r(-T)+\nu(-T)(t+T) \\
& r_{1}(t) \simeq \int_{-T}^{t} \tau \mathrm{d} \tau b\left(r_{0}(\tau)\right) \xi(\tau)
\end{aligned}
$$

Using Eq. (23), we can express the transition PDF $\rho(r(0) \mid \nu(-T), r(-T))$ in Eq. (21) in terms of the equivalent $\mathrm{PDF} \rho\left(r_{1}(0) \mid \nu(-T), r(-T)\right)$ and, using the first of Eq. (23), in terms of the conditional PDF $\rho\left(r_{1}(0) \mid \nu(-T), r_{0}(0)\right)$. Substituting into Eq. (21), with $\rho(r ; 0)=\rho(r)$ and $\rho\left(\nu^{\prime}, r^{\prime} ;-T\right) \simeq \rho_{0}\left(\nu^{\prime}, r^{\prime}\right)=\Omega^{-1} \rho_{0}\left(\nu^{\prime}\right)$, we obtain:

$$
\begin{aligned}
& \rho(r)=\Omega^{-1} \int \mathrm{d} r_{1}(0) \mathrm{d} \nu(-T) \rho_{0}(\nu(-T)) \\
& \quad \times\left.\rho\left(r_{1}(0) \mid \nu(-T), r_{0}(0)\right)\right|_{r_{0}(0)=r-r_{1}(0)},
\end{aligned}
$$

where use has been made of the relation $\rho(r)=$ $\int \mathrm{d} r_{0}(0) \mathrm{d} r_{1}(0) \rho\left(r_{0}(0), r_{1}(0)\right) \delta\left(r_{0}(0)+r_{1}(0)-r\right)$. We can Taylor expand Eq. (24) around $r_{0}(0)=r$ and we obtain

$$
\rho(r) \simeq \frac{1}{2 \Omega} \partial_{r}^{2}\left\langle r_{1}^{2}(0) \mid r_{0}(0)=r\right\rangle,
$$

where the average is on $\nu(-T)$ (in terms of $\left.\rho_{0}\right)$ and $\xi(\tau)$. Using Eqs. (23) and (9), we can write:

$$
\rho(r) \simeq \frac{1}{2 \Omega} \partial_{r}^{2}\left\langle\int_{-T}^{0} \tau^{2} \mathrm{~d} \tau B(r+\nu \tau)\right\rangle,
$$

where the remaining average is on $\nu$; setting $r=0$, we see from Eq. (9) that the time integral is dominated by $\min \left(T, r_{\mathrm{v}} /|\nu|\right)$ and we obtain the final result

$$
\rho(r=0) \sim \Omega^{-1} r_{\mathrm{v}}^{-2} B(0)\left\langle\left(r_{\mathrm{v}} /|\nu|\right)^{3}|| \nu \mid>r_{\mathrm{v}} / T\right\rangle,
$$

where the conditional average to right hand side is peaked at $|\nu| \sim r_{\mathrm{v}} / T$ and diverges for $\epsilon, T / \tau_{S} \rightarrow \infty$.

Thus, ballistic pairs do not dominate the dynamics. A calculation taking into account small velocities in consistent way [34], in turn, would lead to convergent expressions for $\rho(\nu, r)$ and allows to show that the contribution to concentration fluctuation is concentrated at $\nu \sim \nu_{\mathrm{v}} \sim \epsilon^{-1 / 6} \sigma_{v}$, as could have been guessed from the analysis in Section III.

\section{CONCLUSION}

The large Stokes number limit of inertial particles in a random velocity field is plagued by several subtleties. As pointed out in [19, 20], when this limit is reached, the particle dynamics becomes dependent on a new parameter defined in terms of the Stokes and Kubo numbers $S$ and $K$ [see Eq. (3)], namely: $\epsilon=K^{2} S$. This becomes apparent in the case of short-time correlated velocity fields, such as the Kraichnan model 35], in which $K=0$, and the role of the Stokes number is played indeed by $\epsilon$ [from Eq. (3), $S$ is trivially equal to infinity]. Thus, as pointed out in [17], the large Stokes number asymptotics is really the large $\epsilon$ one of Eq. (10).

The point of this paper is that, for $\epsilon \gg 1$, the concentration dynamics is not governed by particle pairs staying close long enough for a local theory on the lines of [15, 21, 31] to work. While, for small $\epsilon$, the particle phase is locally mono-disperse in velocity [36], for $\epsilon>1$, the particle velocity distribution has finite width and only very few such almost co-moving particle pairs exist (see 
discussion at the end of Section III). This property is expected to hold also in more than 1D.

Small relative velocities still appear to be important: as suggested in 28], the particles behave like a gas in thermodynamic equilibrium, but the correlations originate, rather than from random collisions, from particle encounters at small relative velocities, as made clear by the analysis leading to Eq. (25). A qualitative argument in Section III, confirmed by the analysis in [34], suggests that the only particle pairs that have a chance to be correlated are those travelling at relative velocity $\sim \epsilon^{-1 / 6} \sigma_{v}$, that is the minimum for ballistic relative motion at scale $r_{\mathrm{v}}$. However, these velocities are still $O\left(\exp \left(\epsilon^{1 / 3}\right)\right)$ larger than those producing the long time dynamics needed for clustering in the $\epsilon \ll 1$ limit.

The concentration fluctuation variance appears to decay rather slowly, like $\epsilon^{-1 / 2}$ at large $\epsilon$ [see Eq. (20)]. This decay rate could be interpreted as the product of the concentration fraction $\sim \epsilon^{-1 / 6}$ of the particles staying close long enough for their relative velocity to be modified in some way, and their permanence time $\sim \epsilon^{-1 / 3}$ (in units $\left.\tau_{S}\right)$ at separation $|r|<r_{\mathrm{v}}$ [see Eq. (12)].

This mechanism of concentration fluctuation production leads to an important modification of the relative velocity distribution. As particle clusters are the result of particle pairs with small relative velocities, the total velocity PDF will be weighed more to small relative velocities. This results in a depletion of the relative velocity variance, quantified in the exact relation (17), that becomes substantial at $\epsilon \sim 1$. It is to be ascertained whether this property is preserved in more than 1D.

Summarizing, we have the following situation: extrapolating the small $\epsilon$ theory of $[21,26,27]$ to $\epsilon \gtrsim 1$, predicts that clusters become less and less singular, making eventually a transition from fractal to space-filling objects. Extrapolating the opposite way around, the present large $\epsilon$ theory predicts "residual" (not singular) concentration fluctuations, becoming of the order of the mean concentration as $\epsilon \rightarrow 1$, due to more and more particle pairs contributing to the fluctuations. It is reasonable to expect that the situation at $\epsilon \sim 1$ will be a combination of the two pictures.

In [4, 27], it was suggested that clustering is not central in determining collision rates of relevance e.g. in rain formation, as instead are caustics formation and a finite width of the velocity distribution. If the present picture continues to hold in more than $1 \mathrm{D}$, it would result in an even stronger statement, that is, at large $\epsilon$, clustering and modifications to the velocity distribution work against one another. This is in some way analogous to the situation at $\epsilon \rightarrow 0$, of clustering dominance accompanied by caustics disappearance and vanishing of collision rates [21] (see also [37]).

Another feature of concentration fluctuations at large $\epsilon$, is the strong scale separation between their decay length and the correlation length of the fluid: the first is by a factor $\sim \epsilon^{1 / 2}$ larger than the second [see Eq. (20)]. The concentration fluctuation decay length is basically the distance travelled by a particle in a Stokes time and is independent of the fluid correlation structure. Taking into account also the $\epsilon^{-1 / 2}$ variance decay, smaller vortices may thus contribute in a non negligible way to the concentration fluctuations of particles with Stokes time in the turbulent inertial range.

\section{APPENDIX A: FINITE STOKES NUMBER CORRECTIONS}

The Langevin approximation to Eq. (2) provided by (5) is the lowest order in an expansion in inverse powers of the Stokes number $S$. Let us calculate the next order. Given initial conditions at $t=0$, Eq. (2) can be integrated to give:

$$
v(t)=v(0) \mathrm{e}^{-t}+\int_{0}^{t} \mathrm{~d} t^{\prime} \mathrm{e}^{t^{\prime}-t} u\left(x\left(t^{\prime}\right), t^{\prime}\right),
$$

and we can write $x(t)=\bar{x}(t)+\tilde{x}(t)$ with

$$
\bar{x}(t)=x(0)+v(0)\left(1-\mathrm{e}^{-t}\right)
$$

and

$$
\tilde{x}(t)=\int_{0}^{t} \mathrm{~d} t^{\prime}\left(1-\mathrm{e}^{t^{\prime}-t}\right) u\left(x\left(t^{\prime}\right), t^{\prime}\right) .
$$

Following an approach similar to [6], the perturbation expansion is carried on with respect to $\tilde{x}$ and the first correction to $v(t)$ is given by, for $t \ll \tau_{S}=1$ :

$$
\begin{aligned}
& v(t)=v(0)(1-t)+\int_{0}^{t} \mathrm{~d} t^{\prime} u\left(\bar{x}\left(t^{\prime}\right), t^{\prime}\right) \\
& +\int_{0}^{t} \mathrm{~d} t^{\prime} \int_{0}^{t^{\prime}} \mathrm{d} t^{\prime \prime}\left(t^{\prime}-t^{\prime \prime}\right) u^{\prime}\left(\bar{x}\left(t^{\prime}\right), t^{\prime}\right) u\left(\bar{x}\left(t^{\prime \prime}\right), t^{\prime \prime}\right)
\end{aligned}
$$

where $u^{\prime}(x, t)=\partial_{x} u(x, t)$ and $\bar{x}(t) \simeq x(0)+v(0) t$. Taking $t \gg \tau_{E}$, we find that the second piece to right hand side of Eq. (A1) behaves like a Wiener increment; using Eq. (6):

$$
\left\langle\left[\int_{0}^{t} \mathrm{~d} t^{\prime} u\left(\bar{x}\left(t^{\prime}\right), t^{\prime}\right)\right]^{2}\right\rangle=2 \tau_{p}(v(0)) t
$$

and we recover Eq. (5). Notice that the substitution $x\left(t^{\prime}\right) \rightarrow \bar{x}\left(t^{\prime}\right)$ avoids a definition of the noise in implicit form and $\xi(t)$ in Eq. (5) is automatically defined in the Itô prescription [38].

The last term in Eq. (A1) contains a drift correction in the form, from Eq. (11):

$$
\begin{aligned}
& \left\langle u^{\prime}\left(\bar{x}\left(t^{\prime}\right), t^{\prime}\right) u\left(\bar{x}\left(t^{\prime \prime}\right), t^{\prime \prime}\right)\right\rangle=-(K S)^{2} v(0)\left(t^{\prime}-t^{\prime \prime}\right) \\
& \times\left\langle u\left(v(0)\left(t^{\prime}-t^{\prime \prime}\right), t^{\prime}-t^{\prime \prime}\right) u(0,0)\right\rangle .
\end{aligned}
$$

Equation (A1) takes then the final form, in the limit $t \rightarrow$ $\mathrm{d} t$ :

$$
\mathrm{d} v=-[1+\gamma(v)] v \mathrm{~d} t+\left(2 \tau_{p}(v)\right)^{1 / 2} \mathrm{~d} w
$$

where

$$
\gamma(v)=(K S)^{2} \int_{0}^{\infty} \mathrm{d} t t^{2}\langle u(v t, t) u(0,0)\rangle .
$$


and $w(t)=\int_{0}^{t} \xi(\tau) \mathrm{d} \tau$ is the Wiener increment. The noise amplitude is defined up to $O\left(\mathrm{~d} t^{1 / 2}\right)$ terms associated with the fluctuating part of the second line of Eq. (A1).

Thus, the first order correction to the Langevin equation for $v(t)$ is an $O\left(K^{2} / S\right)$ renormalization of the Stokes time, whose sign depends on the profile of the correlation function for $u(x(t), t)$ : a strictly positive correlation corresponds to a decrease of the Stokes time, while oscillations in the correlation may lead to an increase. This would lead to a decrease (an increase) of the drift induced on the particle by an external force.

Notice that the drift correction $\gamma$ provided in Eq. (A3) does not necessarily coincide with what would be ex- pected from a change from Stratonovich to Itô prescription [38]. For instance, adopting for the random field correlation the explicit expression $g(x)=\exp \left(-x^{2} / 2\right)$, would lead to twice what would be obtained interpreting the substitution $x\left(t^{\prime}\right) \rightarrow \bar{x}\left(t^{\prime}\right)$ in $\int_{0}^{t} \mathrm{~d} t^{\prime} u\left(\bar{x}\left(t^{\prime}\right), t^{\prime}\right)$ as a Stratonovich to Itô prescription change. Indicating by $\mathrm{d}_{S}$ the Stratonovich differential and writing $b(v)=$ $\left(2 \tau_{p}(v)\right)^{1 / 2}$ :

$$
b(v) \mathrm{d}_{S} w=\frac{1}{2} b(v) b^{\prime}(v) \mathrm{d} t+b(v) \mathrm{d} w
$$

we find in fact, from Eq. (6) : $\frac{1}{2} b(v) b^{\prime}(v)=-\frac{1}{2} \gamma v$.
[1] J.M. Deutsch "Aggregation-disorder transition induced by fluctuating random forces", J. Phys. A: Math. Gen. 18, 1449 (1985)

[2] M. Cencini, J. Bec, L. Biferale, G. Boffetta, A. Celani, A.S. Lanotte, S. Musacchio and F. Toschi "Dynamics and statistics of heavy particles in turbulent flows", JoT 7, 1 (2006)

[3] R.A. Shaw "Particle-turbulence interactions in atmospheric clouds", Annu. Rev. Fluid Mech. 35, 183 (2003)

[4] G. Falkovich, A. Fouxon and M.G. Stepanov, "Acceleration of rain initiation by cloud turbulence", Nature 419, 151 (2002)

[5] J.W. Brooke, T.J. Hanratty, J.B. Mc Laughlin "Freeflight mixing and deposition of aerosols", Phys. Fluids 6, 3404 (1994)

[6] M.R. Maxey "The gravitational settling of aerosol particles in homogeneous turbulence and random flow fields", J. Fluid Mech. 17, 174 (1987)

[7] J.C.H. Fung and J.C. Vassilicos "Inertial particle segregation by turbulence", Phys. Rev. E 68, 046309 (2003)

[8] T. Elperin, N. Kleeorin and I. Rogachevskii "Selfexcitation of inertial particle concentration in turbulent fluid flows", Phys. Rev. Lett. 77, 5373 (1996)

[9] H. Sigurgeirsson and A.M. Stuart "A model for preferential concentration", Phys. Fluids 14, 4352 (2002)

[10] J. Bec and R. Chétrite "Toward a phenomenological approach to the clustering of heavy particles in turbulent flows", e-print nlin/0701033

[11] G. Falkovich, S. Musacchio, L. Piterbarg and M. Vucelja "Inertial particles driven by a telegraph noise", e-print nlin/0703055

[12] M.R. Maxey and J.J. Riley "Equation of motion for a small rigid particle in a non-uniform flow", Phys. Fluids 26, 883 (1983)

[13] J.R. Fessler, J.D. Kulick and J.K. Eaton "Preferential concentration of heavy particles in a turbulent channel flow", Phys. Fluids 5, 3742 (1994)

[14] L.-P. Wang and M.R. Maxey "Settling velocity and concentration distribution of heavy particles in homogeneous isotropic turbulence", J. Fluid Mech. 256, 27 (1993)

[15] E. Balkovsky, G. Falkovich and A. Fouxon "Intermittent distribution of inertial particles in turbulent flows", Phys. Rev. Lett. 86, 2790 (2001)

[16] P. Olla "Particle transport in a random velocity field with Lagrangian statistics" Phys. Rev. E 66, 056304 (2002)
[17] J. Bec, M. Cencini and R. Hillerbrand "Clustering of heavy particles in the inertial range of turbulence", eprint nlin/0606038

[18] J. Bec, L. Biferale, M. Cencini, A. Lanotte, S. Musacchio and F. Toschi "Heavy particle concentration at dissipative and inertial scales", e-print nlin/0608045

[19] B. Mehlig and M. Wilkinson "Coagulation by random velocity fields as a Kramers problem", Phys. Rev. Lett. 92, 250602 (2004)

[20] K. Duncan, B. Mehlig, S. Östlund and M. Wilkinson "Clustering by mixing flows", Phys. Rev. Lett. 95, 240602 (2005)

[21] M. Wilkinson and B. Mehlig "Path coalescence transition and its applications", Phys. Rev. E 68, 040101(R) (2003)

[22] J. Bec, L. Biferale, M. Cencini, A.S. Lanotte and F. Toschi "On the effects of vortex trapping on the velocity statistics of tracers and heavy particles in turbulent flows", e-print nlin/0604059

[23] J. Bec, L. Biferale, G. Boffetta, A. Celani, M. Cencini, A. Lanotte, S. Musacchio and F. Toschi "Acceleration statistics of heavy particles in turbulence", e-print nlin/0508012

[24] S.A. Derevyanko, G. Falkovich, K. Turitsyn and S. Turitsyn "Explosive growth of inhomogeneities in the distribution of droplets in a turbulent air" JoT (in press) e-print nlin/0602006

[25] M. Wilkinson and B. Mehlig "Caustics in turbulent aerosols", Europhys. Lett. 71, 186 (2005)

[26] J. Bec, M. Cencini and R. Hillerbrand, "Heavy particles in incompressible flows: The large Stokes number asymptotics", Physica D 226, 11 (2007)

[27] M. Wilkinson, B. Mehlig and V. Bezugly "Caustic activation of rain showers", e-print cond-mat/0604166

[28] J. Abrahamson "Collision rates of small particles in a vigorously turbulent fluid", Chem. Eng. Sci. 30, 1371 (1975)

[29] This would be true also if the particles approached with speed $\sigma_{v}$, like in the $\epsilon \gg 1$ case; it is then necessarily true also in the present case, where $|\nu| \ll \sigma_{v}$ (see discussion at the end of the Section).

[30] A simple alternative way to see how caustics disappear for $\epsilon \rightarrow 0$ : from Eq. (13), $\left\langle\hat{\nu}^{2} \mid \hat{r}\right\rangle \sim \hat{r}^{2} \epsilon^{1 / 3}$, while the velocity required for a ballistic flight to $\hat{r}=0$ is $\hat{\nu} \sim$ $\hat{r} / \hat{\tau}_{S} \sim \hat{r} \epsilon^{-1 / 3}$. The probability of such a flight would be: $\sim \exp \left[-\hat{\nu}^{2} /\left(2\left\langle\hat{\nu}^{2} \mid \hat{r}\right\rangle\right)\right] \sim \exp (-c / \epsilon)$, with $c$ a constant. 
[31] L.I. Piterbar "The top Lyapunov exponent for stochastic flow modelling the upper ocean turbulence", SIAM J. Appl. Math. 62, 777 (2002)

[32] L.I. Zaichik and V.M. Alipchenkov "Pair dispersion and preferential concentration of particles in isotropic turbulence", Phys. Fluids 15, 1776 (2003)

[33] J. Zinn-Justin, Quantum Field Theory and Critical Phenomena, 4th edition (Clarendon Press, Oxford 2002)

[34] P. Olla and M.R. Vuolo, " Perturbation theory for large Stokes number particles in random velocity fields" e-print arXiv:0801.3204
[35] R.H. Kraichnan, "Anomalous scaling of a randomly advected passive scalar," Phys. Rev. Lett. 72, 1016 (1994)

[36] Apart near caustics, where the distribution is a superposition of mono-disperse jets.

[37] Y. Zhou,, A.S. Wexler and L.-P. Wang "On the collision rate of small particles in isotropic turbulence. II. Finite inertia case", Phys. Fluids 10, 1206 (1998)

[38] C.W. Gardiner, Handbook for stochastic methods, third edition (Springer NY, 2004) 